\title{
CARACTERÍSTICAS MORFOMÉTRICAS DEL HUEVO FÉRTIL Y SU RELACIÓN CON EL SEXO EN AVES DE POSTURA COMERCIAL MARRÓN
}

\author{
Morphometric characteristics of the fertile egg and its relation to sex in birds with \\ commercial brown posture
}

Artículo de Investigación

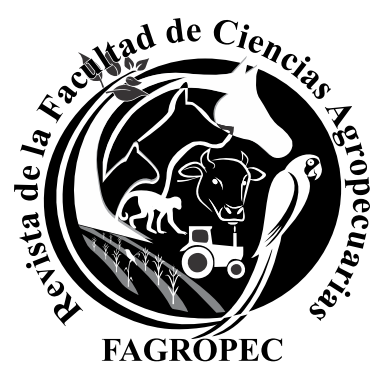

Recibido 21 de agosto de 2019. Aceptado 29 de noviembre de 2019.

Becario Doctoral del Consejo Nacional de Investigaciones Científicas y TécnicasCONICET (Argentina).

(iD) https://orcid.org/0000-0001-7608-2872

2. Instructor Servicio Nacional de Aprendizaje SENA

iD https://orcid.org/0000-0002-3779-7071

Como citar:

MARTÍNEZ MOYANO, Edgar y PINZÓN FERNÁNDEZ, Cesar Augusto. características morfométricas del huevo fértil y su relación con el sexo en aves de postura comercial marrón. En: Revista Facultad Ciencias Agropecuarias FAGROPEC. Universidad de la Amazonia, Florencia - Caquetá. Volumen 12 enero-junio, 2020. Pp. 119-128 ISSN-Revista en Línea: 2539-178X

*Autor para Correspondencia: lizamube@hotmail.com
Edgar Martínez Moyano ${ }^{1 *}$ Ph.D Cesar Augusto Pinzón Fernández ${ }^{2}$ MSc

\section{RESUMEN}

El sector avícola ha sido uno de los subsectores más dinámicos del sector agropecuario en Colombia durante las últimas décadas, sin embargo, la avicultura especializada en la producción del huevo busca encontrar un sistema de sexado económico y de fácil aplicación, para predecir el sexo del ave antes de la eclosión. Dadas esas circunstancias el presente estudio se llevó a cabo como un experimento bajo condiciones controladas para determinar el sexo de 1000 huevos fértiles de aves de postura comercial marrón de la línea Lohmann Brown, con el uso de mediciones morfométricas. Antes de la incubación se midieron el largo, el ancho y la masa del huevo. El volumen y el índice de forma se calcularon utilizando las medidas anteriormente mencionadas. Se encontró que las variables morfométricas ancho, largo e índice de forma del huevo presentaron diferencias estadísticas significativas bajo la prueba LSD de Fisher, en la comparación de medias de los dos sexos $(\mathrm{p}<0.01)$, mientras que el volumen y la masa no presentaron diferencias estadísticas significativas entre los sexos $(\mathrm{p}>0.05)$. El análisis de regresión logística múltiple por pasos hacia adelante evidenció que la variable índice de forma fue la más informativa para captar la probabilidad del sexo del pollito a incubar. De acuerdo con los resultados del presente estudio las mediciones morfológicas del huevo pueden ser un indicador del sexo del pollito, haciendo posible incubar más pollos hembras utilizando las predicciones de estas medidas. Con el fin de disminuir el número de pollos machos que son asesinados en los criaderos y aumentando la capacidad de incubación de pollitos hembra.

Palabras claves:

Sexado; Gallinas Lohmann Brown; Avicultura; Pollos.

\section{ABSTRACT}

The poultry sector has been one of the most dynamic subsectors of the agricultural sector in Colombia during the last decades, however, the aviculture specialized in egg production seeks to find an economical sexing system and easy application, to predict the sex of the bird before hatching Given these circumstances, the present study was carried out as an experiment under controlled conditions to determine the sex of 1000 fertile eggs of commercial brown posture birds of the Lohmann Brown line, with 
the use of morphometric measurements. Before the incubation, the length, width and weight of the egg were measured. The volume and shape index were calculated using the aforementioned measures. It was found that the morphometric variables width, length and egg shape index showed significant statistical differences under the test LSD Fisher, in the comparison of means of the two sexes $(p<0.01)$, while the volume and weight did not present significant statistical differences between the sexes $(\mathrm{p}>0.05)$. The analysis multiple logistic regression by forward steps showed that the shape index variable was the most informative to capture the probability of the sex of the chick to be incubated. According to the results of the present study, the morphological measurements of the egg can be an indicator of the sex of the chick, making it possible to incubate more female chickens using the predictions of these measurements. In order to reduce the number of male chickens that are killed in hatcheries and increasing the capacity of incubation of female chicks.

Key words:

Sexed; Lohmann Brown Hens; Poultry; Chickens.

\section{INTRODUCCIÓN}

El sector avícola sigue creciendo e industrializándose en muchas partes del mundo principalmente por el crecimiento demográfico, el aumento del poder adquisitivo y los procesos de urbanización (FAO, 2019). En Colombia la avicultura fue uno de los grandes protagonistas del crecimiento agropecuario durante el año 2018 (FENAVI, 2018a), constituyendo una alternativa de generación de trabajo y fuente de alimentación para la población. Actualmente la avicultura ocupa el segundo lugar dentro de las actividades agropecuarias en el país después de la ganadería de carne y de leche, y ubicándose por encima de la caficultura (Bohórquez, 2014). Cada día, los colombianos consumen más huevo y más carne de pollo, convirtiendo estas dos proteínas en la base fundamental de su alimentación, el sector en el 2017 tuvo un crecimiento del 6.4 por ciento frente a lo reportado en el 2016(FENAVI, 2018b).

A nivel mundial, Colombia ocupa el sexto lugar en la producción de pollo (después de Estados Unidos, Brasil, México, Canadá y Argentina) y el cuanto en producción de huevo de mesa (después de Estados Unidos, México y Brasil). En las cuentas del gremio, los colombianos consumieron la cifra histórica de 13.827 millones de unidades de huevos en el 2017, lo que arroja un promedio mensual de 1.150 millones de unidades, cifras que ubican al país en el tercer puesto en Latinoamérica, detrás de México y Brasil (FENAVI, 2018b).

La producción tanto de pollo como de huevo se concentra en la región central, integrada por los departamentos de Cundinamarca, Tolima, Huila y Boyacá (32\%), seguida por los Santanderes (25\%), Valle del Cauca (20\%), Antioquia (11\%), Costa Atlántica (7\%) y el eje cafetero (5\%) (Bohórquez, 2014). El consumo per cápita de huevo fue de 279 unidades en el 2017, siete más que el año anterior, lo que le permite al huevo consolidarse como la tercera proteína más consumida en el país, detrás de la carne de pollo y de res; este será muy pronto la segunda proteína más consumida en el país, superando a la carne de res (FENAVI, 2018b). En espera que el sector avícola que continúe creciendo ya que la demanda de carne y huevos es impulsada por la creciente población, los 
crecientes ingresos y la urbanización, el sector se enfrenta a retos sin precedentes.

Actualmente la industria avícola está interesada en métodos rápidos, objetivos y económicos para determinar el sexo de las diferentes aves de importancia comercial tan pronto como sea posible. Su interés reside, principalmente, en obtener hembras productoras de huevos y disminuir el sacrificio a muchos animales machos recién nacidos (González, 2011).

Acorde a lo anterior el sacrificio de aves macho conforma una de mayores problemáticas en las aves de postura comercial, puesto que genera sobrecostos en el sector avícola. De ahí la necesidad de encontrar métodos de identificación del sexo de las aves antes de incubar, siendo este un subsector muy importante en la actividad agropecuaria. Que además sea asequible para pequeños productores y genere confiabilidad en las predicciones.

\section{MATERIALES Y MÉTODOS}

$$
\mathrm{IF}(\%)=\frac{\text { ancho }}{\text { largo }} \times 100
$$

\section{Ubicación:}

El trabajo se desarrolló en la Planta de incubación, PRONAVÍCOLA S.A. - productora avícola

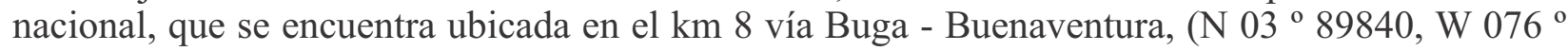
37807) con una altura de 983 msnm (Pronavícola, 2018).

\section{Selección de huevos:}

Se siguió la metodología propuesta por Cobb (2013), adaptada para esta investigación con la selección de 1000 huevos para el estudio, se eligieron huevos con menos de 5 días, ya que después de este tiempo las probabilidades de que el huevo siga fértil baja considerablemente. Los huevos fértiles se proveyeron de un lote de gallinas de postura comercial marrón de la línea Lohmann Brown, criadas bajo condiciones estándar de la empresa Pronavícola S.A., el cual es un sistema tecnificado comercial de gallinas encastadas en piso con una edad de 54 semanas.

\section{Variables:}

Se implementó la metodología propuesta por Morales et al. (2007), adaptada para esta investigación donde cada huevo fue rotulado con numeración consecutiva de 1 al 1000, al cual se le determinó las siguientes medidas morfométricas:

Largo: es el diámetro longitudinal, que corresponde a la dimensión mayor del huevo. Se determinó con un pie de rey con precisión $0.01 \mathrm{~mm}$.

Ancho: es el diámetro transversal, corresponde a la medida que atraviesa la longitud del huevo. Se determinó con un pie de rey precisión $0.01 \mathrm{~mm}$.

Masa: Cada huevo fue pesado en una gramera digital con precisión de $0,1 \mathrm{~g}$.

Indicie de forma: corresponde a la relación entre el largo y ancho del huevo, según Morales et al. (2007) se define mediante la fórmula:

Volumen: el volumen del huevo se calcula utilizando la fórmula:

$$
\mathrm{V}=(\pi / 6) \times \mathrm{L} \times \mathrm{W} 2(\text { Yilmaz y Dikmen, 2013). }
$$




\section{Incubación artificial:}

La incubación se llevó a cabo en una incubadora Chick Master (Genesis IV). Durante este proceso el cual dura 18 días, se tuvieron en cuenta los siguientes parámetros estándar utilizados por la empresa PRONAVICOLAS.A.

- La temperatura de incubación fue de $37.4-37.6^{\circ} \mathrm{C}$

- La humedad relativa durante el proceso debe oscilar entre 81.5 y $85.5 \%$

- La ventilación es constante ya que es necesaria durante la incubación para proporcionar el oxígeno que el embrión va consumiendo y para eliminar el $\mathrm{CO} 2$, el vapor de agua y exceso de temperatura que se produce en su interior. El contenido de CO2 debe estar bajo control, este no debe superar el $0,5 \%$.

- Posición del huevo: Los huevos fueron volteados durante el proceso de incubación. Esto evita que el embrión se pegue a las membranas de la cáscara, particularmente durante los primeros 18 días, la frecuencia de volteo fue de una vez cada 1 o 2 horas, el giro alcanzó los 90 grados y los huevos fueron mantenidos a 45 grados de una vertical imaginaria (Tullett, 2010).

Al día 19 los huevos fueron transferidos a nacedora (bandejas). Este proceso es necesario para que el polluelo, al nacer, tenga más libertad de movimientos y pueda salir del cascarón en una bandeja y no en el alveolo inclinado en que está colocado en la incubadora. La nacedora tuvo una temperatura entre 36,8 y $37,1^{\circ} \mathrm{C}$ y una humedad relativa entre 60 y $80 \%$, según parámetros estándar utilizados por la empresa PRONAVICOLAS.A.

A las bandejas se le realizó divisiones para cada huevo, con el fin de que los polluelos no se mezclen al nacer y poder continuar con la toma de información correspondiente a determinar el sexo.

\section{Sexado de los polluelos:}

El día 21 cada polluelo fue revisado, determinando su sexo por el método de autosexado por color, los machos nacen de color blanco y las hembras de color Brown. Esta metodología es la utilizada por la empresa Pronavicola S.A.S para esta línea de aves. Al finalizar el proceso de incubación fueron sexados 854 aves.

\section{Análisis de datos:}

Las características (variables) morfométricas de los huevos fueron sometidas a estadística descriptiva y análisis de varianza mediante la prueba LSD Fisher $(\mathrm{p}<0.05)$, con el fin de evaluar la fuerza de asociación con la variable dependiente (sexo) mediante la diferencia de medias. Aunado a lo anterior se realizaron regresiones logísticas simples para cada una de las variables evaluadas, con el fin de identificar un modelo, la tendencia y relación de las variables morfométricas con la variable dependiente (sexo).

\section{Desarrollo del modelo de sexaje:}

Para desarrollar el método morfométrico para el sexado de huevos se utilizó una regresión logística por pasos hacia adelante en el software SPSS v. 25, siguiendo la metodología propuesta por Yilmaz y Dikmen (2013); esta regresión se utiliza para estudiar qué factores o covariables modifican y en qué medida la probabilidad de ocurrencia de un suceso (Di Rienzo, 2011). Es decir, permite calcular la probabilidad de que la variable dependiente (Sexo) pertenezca a cada una de las dos categorías (Macho - Hembra) en función del valor que adquiera la variable independiente (ancho, largo, masa, 
índice de forma y volumen).

Se usó regresión logística múltiple, donde se buscó determinar la mejor combinación de variables determinadas (masa, largo, ancho, índice de forma y volumen), que en conjunto permiten obtener la mayor certeza del modelo (Lever et al., 2016).

Además, se analizaron los criterios de sensibilidad y especificidad, el primero que mide cuan capaz es el criterio pronóstico de anticipar o detectar un resultado positivo, el segundo indica cuan acertado es un pronóstico dado (Di Rienzo, 2011); seguidamente se realizó el análisis del área bajo la curva de ROC, que permite evidenciar qué tan bueno es el criterio pronóstico o diagnóstico (Di Rienzo, 2018).

\section{RESULTADOS Y ANÁLISIS}

La prueba LSD de Fisher efectuada para determinar la significancia de las variables morfométricas del huevo fértil sobre el sexo del mismo, en aves de postura comercial marrón evidenció, que en cuanto a la masa $(\mathrm{g})$ y el volumen $\left(\mathrm{mm}^{3}\right)$ no se presentó diferencias estadísticas significativas $(\mathrm{p}>$ 0.05); mientras que para las variables ancho $(\mathrm{mm})$, largo $(\mathrm{mm})$ e índice de forma se presentaron diferencias estadísticas significativas $(\mathrm{p}<0.05)$, lo que indica estas variables presentaron la mayor fuerza de asociación con la variable dependiente (sexo).

Tendencia de las variables morfométricas en la predicción del sexo del pollito hembra para aves de postura comercial marrón.

La curva de probabilidad de ser pollito hembra de acuerdo con la masa ( $\mathrm{g}$ ) del huevo, para valores entre 49 y 75 g (Figura 1), permitió observar que esta variable morfométrica no presentó significación estadística $(\mathrm{p}>0.05)$ como predictora del sexo de los huevos; sin embargo, se pudo evidenciar que la probabilidad de ser pollito hembra aumenta a medida que la masa es mayor.

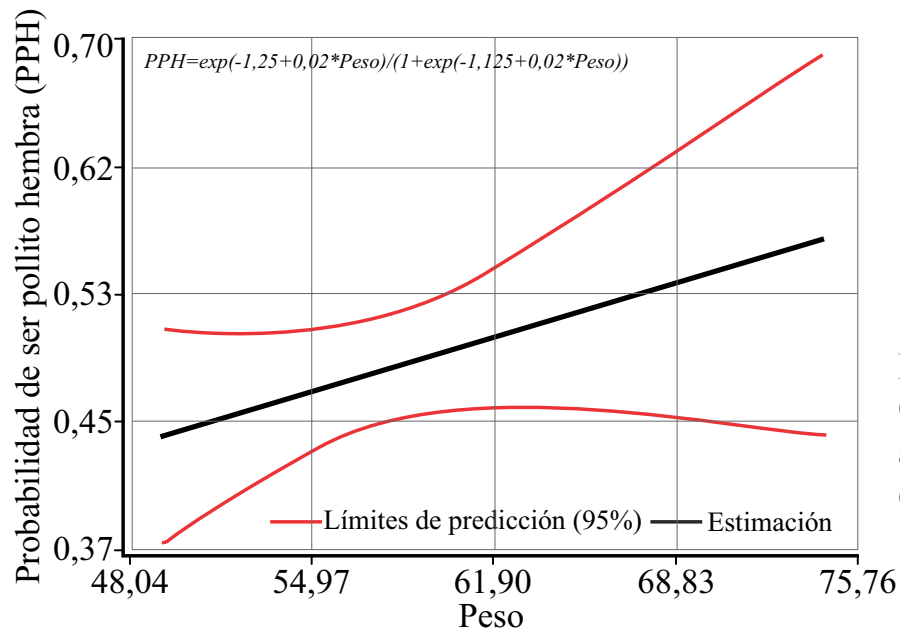

Figura 1.

Curva de probabilidad de ser pollito hembra de acuerdo con la masa $(\mathrm{g})$ del huevo, para valores entre 49 y $75 \mathrm{~g}$.

La curva de probabilidad de ser pollito hembra de acuerdo con el ancho ( $\mathrm{mm}$ ) del huevo, para valores entre 38 y $47 \mathrm{~mm}$ (Figura 2), permitió observar que esta variable morfométrica presentó significancia estadística ( $\mathrm{p}<0.01)$ como predictora del sexo de los huevos; donde se pudo evidenciar que la probabilidad de ser pollito hembra aumenta a medida que el ancho del huevo es mayor. 


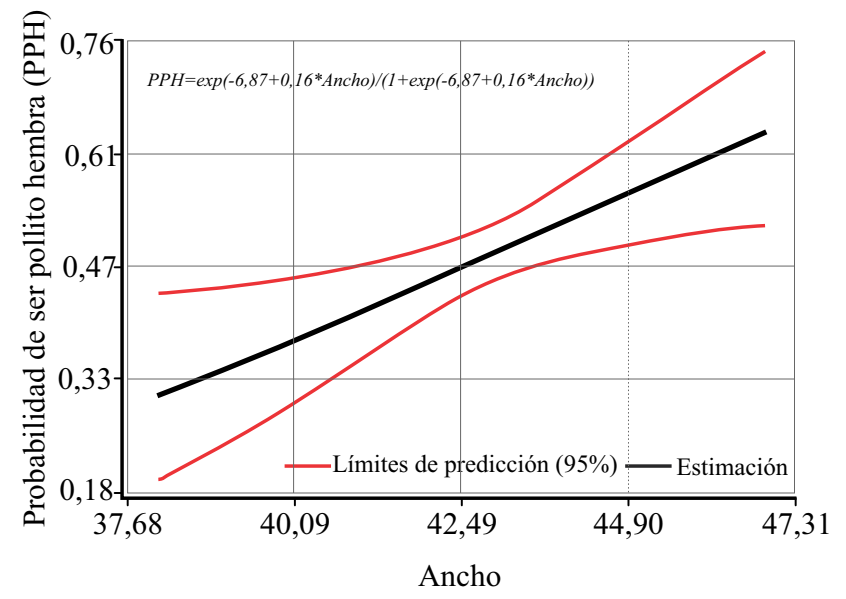

\section{Figura 2.}

Curva de probabilidad de ser pollito hembra de acuerdo con el ancho ( $\mathrm{mm})$ del huevo, para valores entre 38 y $47 \mathrm{~mm}$.

La curva de probabilidad de ser pollito hembra de acuerdo con el largo ( $\mathrm{mm}$ ) del huevo, para valores entre 48 y $62 \mathrm{~mm}$ (Figura 3), permitió observar que esta variable morfométrica presentó significación estadística $(\mathrm{p}<0.01)$ como predictora del sexo de los huevos; donde se pudo evidenciar que la probabilidad de ser pollito hembra disminuye a medida que el largo del huevo es mayor.

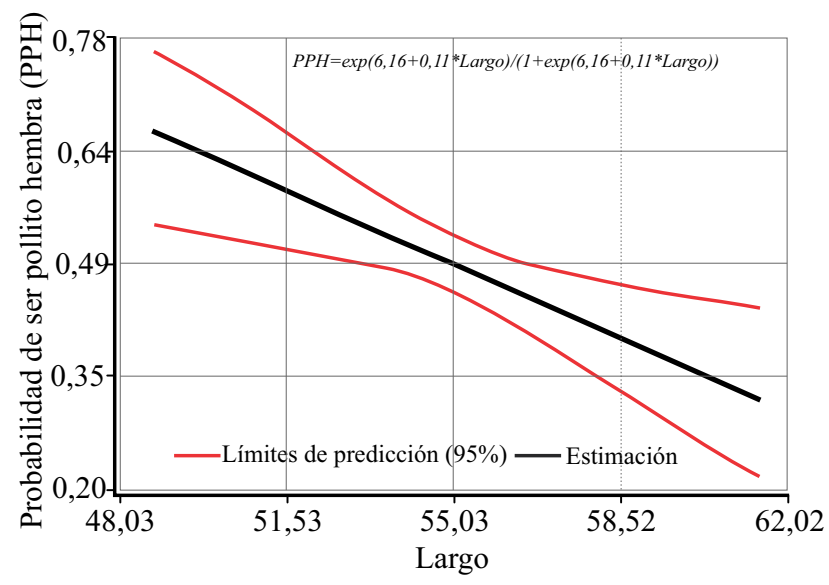

\section{Figura 3.}

Curva de probabilidad de ser pollito hembra de acuerdo con el largo ( $\mathrm{mm}$ ) del huevo, para valores entre 48 y $62 \mathrm{~mm}$.

La curva de probabilidad de ser pollito hembra de acuerdo con el índice de forma (\%) del huevo, para valores entre 68 y $90 \%$ (Figura 4), permitió observar que esta variable morfométrica presentó significación estadística $(\mathrm{p}<0.001)$ como predictora del sexo de los huevos; donde se pudo

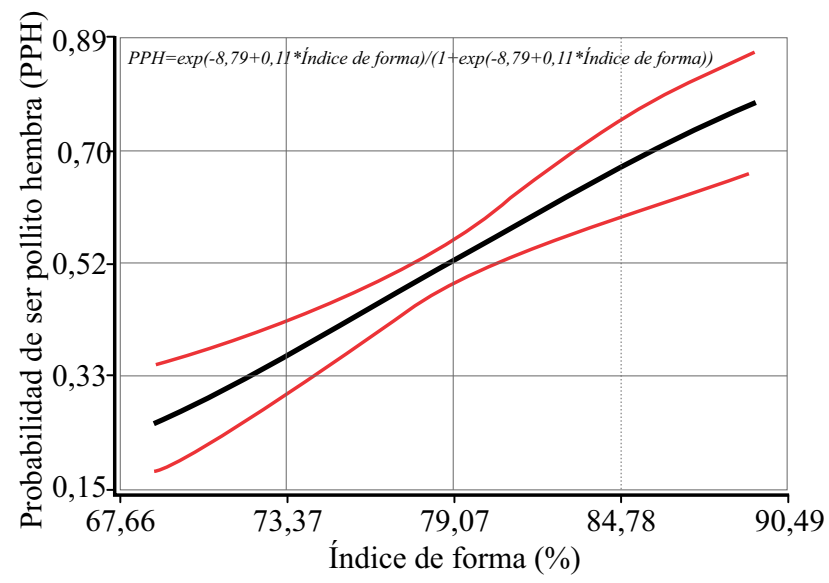

\section{Figura 4.}

Curva de probabilidad de ser pollito hembra de acuerdo con el índice de forma (\%) del huevo, para valores entre 68 y $90 \%$. 
evidenciar que la probabilidad de ser pollito hembra es mayor a medida que el índice de forma del huevo se incrementa.

La curva de probabilidad de ser pollito hembra de acuerdo con el volumen $\left(\mathrm{mm}^{3}\right)$ del huevo, para valores entre 40881 y $67136 \mathrm{~mm}^{3}$ (Figura 5), permitió observar que esta variable morfométrica no presentó significativamente estadística $(\mathrm{p}>0.05)$.

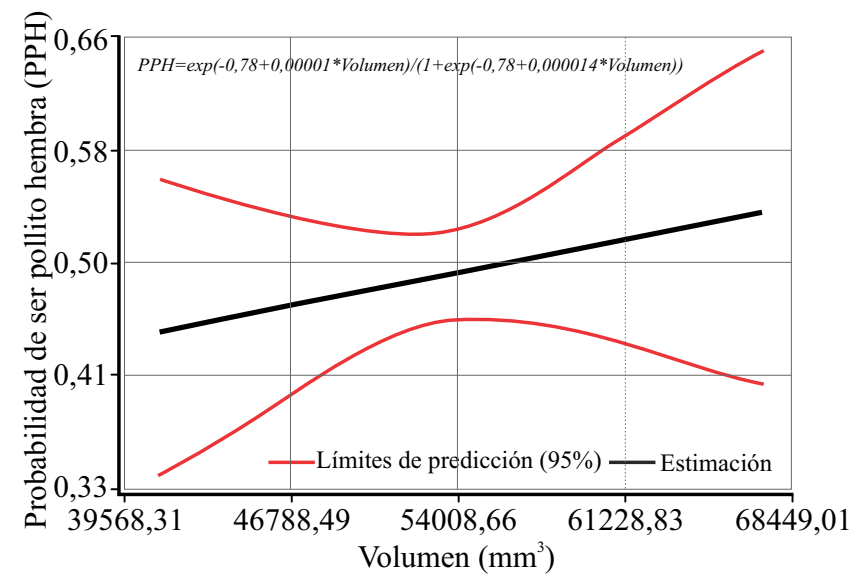

Figura 5.

Curva de probabilidad de ser pollito hembra de acuerdo con el volumen $\left(\mathrm{mm}^{3}\right)$ del huevo, para valores entre 40881 y $67136 \mathrm{~mm}^{3}$.

\section{Desarrollo de un modelo que permita determinar el sexo del huevo para aves de postura comercial marrón.}

Se usaron análisis de regresión logística binaria múltiple por pasos (Yilmaz y Dikmen, 2013) para evaluar la probabilidad de sexo del pollito para incubar, de acuerdo con la significancia estadística ( $p$ $<0.05)$ de las variables índice forma, ancho y largo del huevo. Solo estas mediciones morfológicas fueron probadas para determinar la probabilidad de sexo del pollito para incubar, dado que fueron las variables que presentaron mayor fuerza de asociación con la variable dependiente, mediante la diferencia de medias. El mejor modelo de ajuste para determinar el sexo del pollito de incubación se encontró con el uso de un procedimiento paso a paso hacia adelante en el método de regresión logística múltiple. De acuerdo con el análisis gradual de los factores índice de forma, anchura y longitud; el índice de forma fue el único factor que se mantuvo en el modelo $(\mathrm{p}<0.001)$. El efecto del índice de forma en el sexo del pollito para incubar fue el factor más prominente para determinar el sexo del huevo en aves de postura comercial marrón.

La prueba de bondad de ajuste de Hosmer \& Lemeshow (Hosmer \& Lemeshow, 1989) se encontró en 0.527 para el modelo seleccionado en el método hacia adelante por pasos. Por su parte $-2 \log$ de la verosimilitud o desviación que mide hasta qué punto un modelo se ajusta bien a los datos evidenció 1161.2 con cuatro interacciones; al igual que el R cuadrado de Cox y Snell que es un coeficiente de determinación generalizado, indica que el $2.5 \%$ de la variación de la variable dependiente es explicada por la variable incluida en el modelo, al igual que el R cuadrado de Nagelkerke que indica un 3.4\% de la variación. Coeficientes que presentaron valores bajos y podrían ser mejorados al agregar una nueva variable de evaluación, para un mayor ajuste dentro del modelo.

De acuerdo con los análisis de regresión múltiple por pasos, solo se dejó el índice de forma en el modelo para determinar la probabilidad de ser un pollito hembra, indicando que cuanto más altos 
sean los valores del índice de forma, mayor será la probabilidad de incubar una hembra:

$$
\begin{gathered}
\text { Probabilidad de ser pollito hembra } \\
(\mathrm{PPH})=(\mathrm{PPH})=\operatorname{EXP}(-8,79+0,11 * \mathrm{IF}) /(1+\mathrm{EXP}(-8,79+0,11 * \mathrm{IF}))
\end{gathered}
$$

\section{DISCUSIÓN}

Los resultados de este estudio presentan una relación directa con la variable índice de forma que para este fue la que presentó la mejor significancia estadística $(\mathrm{p}<0.0001)$, lo que indica que los huevos con mayor índice de forma existen mayores probabilidades de que produzca un pollito hembra; esto concuerda con lo obtenido por Yilmarz y Dikmen (2013), quienes encontraron que esta variable es la que presenta la mejor significancia estadística y por tanto quien mejor predice la probabilidad de salir pollitos hembras.

Las variables largo y el ancho fueron significativos pero no aportan mucha información a los resultados obtenidos para este estudio y para la predicción del modelo de regresión, se conoce que el sexo de los huevos en los pollos se determina poco antes de la ovulación (Romanoff, 1960), este proceso se puede observar durante la primera división meiótica, cuando la segregación de los cromosomas sexuales (en aves la hembra es el sexo heterogamético) consigna el cromosoma W o Z al óvulo y al ovocito. Cromosoma sexual restante para el cuerpo polar (Sturkie, 2000).

Cualquiera de estas dos variables, tamaño o forma, podría proporcionar a las aves en incubación un medio simple de "elegir" el sexo de la descendencia negando selectivamente la incubación de algunos huevos. Ligon y Ligon (1990) observaron que los huevos solos de la abubilla arbórea-verde (Phoeniculus purpureus) se alejaban del resto del embrague a un lado de la cavidad del nido, lo que sugiere una incubación selectiva. Es importante recordar que el tamaño relativo y la forma de los huevos producidos están, activa o pasivamente, bajo control materno y pueden proporcionar un mecanismo de detección de sexo para especies que no pueden manipularse antes de la puesta (Pike y Petrie, 2003).

Las variables largo y ancho de los huevos fueron significativas para este estudio $(\mathrm{p}<0.05)$ pero, no fueron tan útiles para la predicción del modelo de predicción; donde el análisis por pares de huevos masculinos y femeninos dentro del mismo embrague indicó que el sexo del embrión explicó el 7\% de la varianza para el largo relativo del huevo (Cordero et al., 2000).

La probabilidad de incubar un pollito femenino se redujo con el aumento de la longitud del huevo para incubar ( $<<0.05)$. Similar a los hallazgos de Cordero et al. (2000), quienes demostraron que, en los huevos de gorrión en casa, aunque el ancho del huevo no estaba relacionado con el sexo del huevo, los huevos masculinos eran significativamente más largos que los huevos femeninos. Burnham et al. (2003) encontraron que el largo y el ancho del huevo del halcón peregrino eran malos predictores del sexo de los pollitos.

Cordero et al. (2000), informaron que en el estornino inmaculado (Sturnus unicolor) los huevos hembra eran más pesados que los huevos machos y estas diferencias eran independientes de la secuencia de puesta porque la interacción entre el sexo y el orden de puesta no era significativa. En el presente estudio las probabilidades de tener un pollito hembra no se vieron afectadas por la masa del 
huevo antes de la incubación ( $\mathrm{p}>0.05)$. De manera similar, Rutkowska y Cichon (2005) encontraron que el sexo de los descendientes de los pinzones cebra no estaba relacionado con la masa del huevo. Sagar et al. (2005) encontraron que en el albatros de Buller (Thalassarche bulleri) el tamaño del huevo no tuvo una influencia detectable en el sexo resultante del pollito.

\section{CONCLUSIÓN}

Finalmente, se concluye que los métodos morfométricos para el sexado de huevos en gallinas de línea Lohmann Brown, representan un impacto importante en la gestión del proceso de incubación en las empresas avícolas, permitiendo que a través de estas medidas morfométricas se puedan incubar más pollos hembras; con el fin de aumentar la capacidad de incubación y disminuir la cantidad de pollos machos que se matan en los criaderos.

El análisis de las curvas de sensibilidad y especificidad, al igual que la curva de ROC son criterios importantes para evaluar el ajuste y la capacidad predictiva del criterio del modelo; las primeras permiten identificar el umbral en el cual las predicciones presentan su mayor capacidad, mientras que la curva de ROC ostenta la capacidad pronóstica del modelo. Teniendo en cuenta el índice de forma que fue la variable más representativa, se creó un modelo con capacidad predictora del 59\%.

\section{LITERATURA CITADA}

BOHÓRQUEZ, V. Perspectiva de la Producción Avícola en Colombia. Universidad Militar Nueva Granada, Bogotá. 2014.Pp. 30

BURNHAM, W., SANDFORT, C., \& BELTHOFF, J. R. Peregrine Falcon eggs: egg size, hatchling sex, and clutch sex ratios. The Condor, 105(2). 2003. Pp. 327-335.

CORDERO, P. J., GRIFFITH, S. C., APARICIO, J. M., \& PARKIN, D. T. Sexual dimorphism in house sparrow eggs. Behavioral Ecology and Sociobiology, 48(5). 2000. Pp. 353-357.

DI RIENZO J.A., CASANOVES F., BALZARINI M.G., GONZALEZ L., TABLADA M., \& ROBLEDO C.W. InfoStat versión.Grupo InfoStat, FCA, Universidad Nacional de Córdoba, Argentina. 2018. \{En línea\}. Disponible en: https://cutt.ly/UyIr1A1

DI RIENZO, J.A. Análisis de Regresión Logística. Grupo InfoStat, FCA., Universidad Nacional de Córdoba. 2011

FAO.Producción y productos avícolas. 2019. \{En línea\}. Disponible en: https://cutt.ly/iyIrkAN

FENAVI. El sector avícola en Colombia creció 4.5\% en 2018. (2018a). \{En línea\}. Disponible en: https://cutt.ly/OyIrxVQ

FENAVI. Fondo Nacional Avícola-Estadísticas. (2018b). \{En línea $\}$. Disponible en: https://cutt.ly/9yIrnQV

GONZALES, B. Un nuevo método de sexado de aves evitará el sacrificio de muchos machos. Artículos aves. Informative veterinario Albéitar.2011 
HOSMER, D. W., \& LEMESHOW, S. Applied Logistic Regression. New York: John Wiley \& Sons. 1989.

LEVER, J., KRZYWINSKI, M., \& ALTMAN, N. Puntos de significación: regresión logística. 2016

LIGON, J.D., \& LIGON, S.H. The communal social system of the green woodhoopoe in Kenya. Living Bird, 17. 1978. Pp. 159-197.

MORALES, J., DUARTE, M., \& ZÚÑIGA, H. Caracterización físico-química del huevo del caimán llanero, Crocodylus intermedius, Graves 1819. Acta zoológica mexicana, 23(3). 2007. Pp. 17-27.

PIKE T.W., \& PETRIE, M. Potential mechanisms of avian sex manipulation. Biological Review, 78. 2003. Pp. 553-574.

PRONAVÍCOLA. Localización. 2018. \{En línea\}. Disponible en: https://cutt.ly/8yIrT69

ROMANOFF, A.J. The avian embryo, structural and functional development. New York: The Macmillian Company. 1960

RUTKOWSKA, J., \& CICHON, M. Egg size, offspring sex and hatching asynchrony in zebra finches Taeniopygia guttata. Journal Avian Biology, 36. 2005. Pp.12-17.

SAGAR, P. M., UNWIN, M.J, STAHL, J.C., \& WARHAM, J. Variation in the size of Buller's albatross (Thalassarche bulleri) eggs. New Zealand J. Zoology, 32. 2005. Pp.171-180.

STURKIE, P.D. Sturkie's avian physiology. 5th ed. San Diego: Academic Press. 2000

TULLETT, S. Investigación de las prácticas de incubación. ROSS. 2010. Pp. 48

YILMAZ, B., \& DIKMEN, S. A morphometric method of sexing white layer eggs. Revista Brasileira de Ciência Avícola, 15(3). 2013. Pp. 203-210. 